\title{
Indefinite Problem in Wasan
}

\author{
和算家的累约术
}

QU Anjing 曲安京

\begin{abstract}
Japanese mathematics, namely Wasan, was well-developed before the Meiji period. Takebe Katahiro (1664-1739)and Nakane Genkei (1662-1733) , among a great number of mathematicians in Wasan, maybe the most famous ones. Taking Takebe and Nakane's indefinite problems as examples, the similarities and differences are made between Wasan and Chinese mathematics. According to investigating the sources and attitudes to these problems which both Japanese and Chinese mathematicians dealt with, the paper tries to show how and why Japanese mathematicians accepted Chinese tradition and beyond. As a typical sample of the succession of Chinese tradition, Wasan will help people to understand the real meaning of Chinese tradition deeper.
\end{abstract}

Keywords: Wasan, indefinite problem, Takebe Katahiro, Nakane Genkei; 和算, 不定 分析，建部贤弘，中根元珪.

MSC: 01

\section{1 序论}

日本传统数学 (和算) 在 17 世纪中叶出现了一大批著名的数学家和数学成果。矢 孝和（Seki Takakazu 1642?-1708）及其弟子建部贤弘（Takebe Katahiro 1664-1739） 是其代表人物。

和算的成长时期, 大体与我国的清代同时,他们是在接受传统中国数学的基础上发 展起来的。与清代数学家不同的是, 和算家研究的问题, 虽然大部分都可以追朔到中算 的源头, 但是, 这些问题, 基本上都被和算家抽象为纯粹的数学问题, 且多有创造性的贡 献。而与和算家同时代的明清数学家, 则在对传统数学的继承与创新方面, 相较和算家 逊色很多。

国家自然科学基金（编号 : 10771169）

承小川束教授惠赠电子版《累约术》文件，在此敬致谢忱。

QU Anjing: Dept. of Math., Northwest Univ., Xian, China E-mail: qaj@nwu.edu.cn Receiveded on Nov. 3, 2013, revised on Nov. 22, 2013, accepted on Nov. 28, 2013. 
比较和算与中算的异同, 对于我们理解明清时期复兴中国传统数学的各种努力,特 别是对我们理解中国古代数学的机械化程序算法体系的特征, 及其在整个数学史长河 中的地位, 是有重要意义的。

建部贤弘和中根元珪 (Nakane Genkei 1662-1733) 的累约术, 基本上根源于中国传 统数理天文学中提出的问题, 建部贤弘的《累约术》的算法思想, 前人已经有不少的研 究，而中根元珪的《累约拾遗》，可能是涉及到了具体的历法问题，比较艰涩，因此，少有 数学史家对其进行深入的分析。

本文以和算家的累约术为例，通过探讨这些问题的来源，特别是对中根元珪有矢历 算问题的造术原理进行了详细的讨论，在复原和算家处理这些问题的思想方法的基础 上, 比较其与中国古代算法的异同, 以期从一个专门的视角, 说明和算家对中国传统数 学的机械化程序算法体系的继承与创新。

\section{2 中日不定分析问题的来源}

建部贤弘的《累约术》与中根元珪的《累约拾遗》，是和算家处理一次不定不等式或 一次同余式的算法。这两部著作的抄本在日本的一些学术机构中可以找到。[6] 建部 贤弘《累约术》的问题表述与解法均为一次不定不等式; 中根元珪的《累约拾遗》，则 用同余式的方式，处理相矢的问题。

建部贤弘的《累约术》是一篇 12 页的短文，由三个问题及其解法组成。[6]他所考 虑的主要问题, 可以表述为如下形式的不定不等式：

$$
|a x-b y-c|<1
$$

其中 $a 、 b 、 c$ 为给定的有理数。中根元珪的《累约拾遗》则是由三个问题和两个历算问 题的应用及其解法构成。[6] 我们将会看到, 中根元珪将所有问题都化为如下的标准的 同余形式：

$$
a x-c \equiv r \quad(\bmod b)
$$

其中 $0<r<d_{\text {。 }}$ 不难看出，不定不等式（1）与同余式（2）彼此可以转换。

《累约拾遗》的两个应用, 都是根据日本当时行用的中国历法《宣明历》（822）而设 计的问题, 可以肯定地说, 《累约拾遗》就是为了解决这些历算问题而专门撰著的。

而在中国古代, 为了计算上元积年, 历法家们发明了所谓的『演纪术』。简单地说， 上元, 是一种特殊的历元, 基本上是所有历法项目的共同起算点, 为了计算这个历元, 需 要求解一个同余式组。可惜的是，在《授时历》（1280）废除演纪上元之后，明清时期的 数学家已经无人知晓推算上元的具体程序。理论上讲, 可以用中国剩余定理解决这个 问题，但实际上，中国古代的历法家是按照代入法，依次逐个求解同余式，以获得理想的 
上元。[4] 这个算法, 被称为《演纪术》。对于演纪术, 只需要知道如下同余式的解法

$$
a x \equiv r^{\prime}(\bmod b)
$$

其中 $c<r^{\prime}<d_{\text {。 }}$ 若令 $r=r^{\prime}-c$, 则 (3) 式立刻转化为中根元珪的同余式 (2)。

由此可见，和算家的累约术的问题来源，与中国古代的同余理论类似，大体上都是 源自历法中的实际问题。

3 建部贤弘的累约术

为了得到不定不等式 (1) 的一对整数解 $(x, y)$, 建部贤弘首先考虑 $c=0$ 的情形。 藤原松三郎（1881-1941）曾经对建部贤弘的累约术进行过详细的讨论。[8]

\section{1 建部贤弘的连分数算法}

根据藤原的解读, 建部贤弘是按照如下的方式处理不定不等式

$$
|a x-b y|<1
$$

首先, 按照连分数展开, 可以得到如下的序列：

$$
\frac{b}{a}=a_{1}+\frac{r_{1}}{a}, \quad \frac{a}{r_{1}}=a_{2}+\frac{r_{2}}{r_{1}}, \quad \frac{r_{1}}{r_{2}}=a_{3}+\frac{r_{3}}{r_{2}}, \quad \cdots, \quad \frac{r_{n-2}}{r_{n-1}}=a_{n}+\frac{r_{n}}{r_{n-1}}
$$

根据这些展式所得到的商 $a_{n}$ 与不尽 $r_{n}$, 可以获得 $b / a$ 的一系列渐近分数 $p_{n} / q_{n}$, 其算法 程序可以用表格表示出来, 如表 1 所示。

根据表 1 给出的程序，可以得到如下的䏌系式：

$$
a p_{n}-b q_{n}=(-1)^{n} r_{n}
$$

当 $r_{n}<1$ 时, 就可以得到满足不定不等式 (11) 的一对整数解 :

$$
(x, y)=\left(p_{n}, q_{n}\right)
$$

显而易见, 建部贤弘给出的求解 $\left(p_{n}, q_{n}\right)$ 的程序, 与按连分数展开求渐近分数的程序是 一致的。

\begin{tabular}{cccc}
\hline 商 & 不尽 & 益段 & 损段 \\
\hline$a_{1}$ & $r_{1}=b-a a_{1}$ & $p_{1}=a_{1}$ & $q_{1}=1$ \\
$a_{2}$ & $r_{2}=a-r_{1} a_{2}$ & $p_{2}=1+p_{1} a_{2}$ & $q_{2}=a_{2}$ \\
$a_{3}$ & $r_{3}=r_{1}-r_{2} a_{3}$ & $p_{3}=p_{1}+p_{2} a_{3}$ & $q_{3}=q_{1}+q_{2} a_{3}$ \\
$a_{4}$ & $r_{4}=r_{2}-r_{3} a_{4}$ & $p_{4}=p_{2}+p_{3} a_{4}$ & $q_{4}=q_{2}+q_{3} a_{4}$ \\
$\ldots$ & $\ldots$ & $\ldots$ & $\ldots$ \\
$a_{n}$ & $r_{n}=r_{n-2}-r_{n-1} a_{n}$ & $p_{n}=p_{n-2}+p_{n-1} a_{n}$ & $q_{n}=q_{n-2}+q_{n-1} a_{n}$ \\
\hline
\end{tabular}

Table 1. The iteration process of successive divisions; 累约术之连分数程序

《累约术》设计的第一问, 专门说明上述程序的应用。原题称： 
假如有以一千三百一十八个七七○六累益之, 以五万九千五百九十四个七 七○五累损之,殚个数, 问强弱损益段各几何?

答曰：弱益段：四千 $\bigcirc$ 二十；弱损段：八十九。强益段：五十五万○ ○六十五；强损段：一万二千一百七十二。

根据上述文字, 可以列出如下的不定不等式：

$$
|1318.7306 x-59594.7705 y|<1
$$

假设 $\left(p_{n}, q_{n}\right)$ 是一组解，所谓『弱损㿽段』，是指 $\left(p_{n}, q_{n}\right)$ 满足

$$
-1<a p_{n}-b q_{n}<0
$$

『强损益段』，是指 $\left(p_{n}, q_{n}\right)$ 满足

$$
0<a p_{n}-b q_{n}<1
$$

《累约术》给出上述问题的演草如表 2 所示。

\begin{tabular}{ccccc}
\hline 号 $n$ & 商 $a_{n}$ & 不尽 $r_{n}$ & 益段 $p_{n}$ & 损段 $q_{n}$ \\
\hline 甲 1 & 45 & 弱 251.8935 & 45 & 1 \\
乙 2 & 3 & 强 59.2631 & 226 & 5 \\
丙 3 & 4 & 弱 14.8411 & 949 & 21 \\
丁 4 & 2 & 强 14.7398 & 3073 & 68 \\
戊 5 & 1 & 弱 0.1013 & 4022 & 89 \\
己 6 & 136 & 强 0.9630 & 550065 & 12172 \\
\hline
\end{tabular}

Table 2. The description of the 1st problem in the Method of Successive Divisions; 《累约术》第 一问演草

这个程序中求序列 $\left\{p_{n}\right\}$ 的部分, 就是中国数学史上著名的大衍求一术。（5）式也 可以表示成

$$
a p_{n}=(-1)^{n} r_{n} \quad(\bmod b)
$$

当 $b, a$ 都是整数的时候，总是可以得到 $(-1)^{n} r_{n}=1$, 此时 $p_{n}=k$ 就是我们所熟悉的乘 率, 它使得

$$
a k \equiv 1 \quad(\bmod b)
$$

据此, 可以得到同余式 (3) 的解。不过, 在中国数学史上, 我们没有发现求损段序列 $\left\{q_{n}\right\}$ 的程序，因此，矢于是否中国古代已经有连分数算法的猜测，始终没有得到肯定。[1,2]

\section{2 建部贤弘的累约术是如何构造出来的?}

建部贤弘累约术的数学意义, 是藤原松三郎首先揭示的,他用现代数学语言对累约 术的算法程序进行了详细的解释, 这些工作已经写入了日本数学史, 而广为人知。[3] 
我们知道, 建部贤弘的累约术, 目的在于求解形如（1）式的不定不等式, 这个不等 式,也可以写为如下形式的不定方程：

$$
a x-b y-c=r, \quad|r|<1
$$

其中： $0<a<b, c$ 为任意给定的有理数。我们感兴趣的是, 建部贤弘到底是如何构 造出他的累约术的? 这是本节所要讨论的主要问题。

前面已经提到, 为了求解形如（6）式的不定方程, 建部贤弘利用连分数展开的方 法, 首先构造出了当 $c=0$ 时的相矢算法, 并得到䏌系式（5）:

$$
a p_{n}-b q_{n}=(-1)^{n} r_{n}
$$

这个结果非常重要。建部贤弘由此出发, 构造不定方程（6）的算法程序。首先, 由

$$
\frac{b+c}{a}=b_{1}-\frac{s_{1}}{a}, \quad s_{1}<a
$$

可以得到

$$
a b_{1}-b-c=s_{1}
$$

若令 $x_{1}=b_{1}, y_{1}=1$, 上式可以写成如下的形式：

$$
a x_{1}-b y_{1}-c=s_{1}
$$

比较（5）与（6）, 不难知道，如果我们可以找到一系列的 $s_{n}$, 使之不断地缩小, 直至小 于 1 , 则对应的 $x_{n}$ 与 $y_{n}$ ，就可以满足不定方程（5）。由于序列 $\left\{r_{n}\right\}$ 是逐次递减的，因 此，可以利用 $r_{n}$ ，来构造 $s_{n 。}$ 所以，令

$$
\frac{s_{1}}{r_{1}}=b_{2}-\frac{s_{2}}{r_{1}}, \quad s_{2}<r_{1}
$$

由此可得

$$
s_{2}=r_{1} b_{2}-s_{1}
$$

根据（5）式，可以得到

$$
a p_{1} b_{2}-b q_{1} b_{2}=-r_{1} b_{2}
$$

相加（7）与 (8)，可以得到

$$
a\left(x_{1}+p_{1} b_{2}\right)-b\left(y_{1}+q_{1} b_{2}\right)-c=-\left(r_{1} b_{2}-s_{1}\right)
$$

若令 $x_{2}=x_{1}+p_{1} b_{2}, y_{2}=y_{1}+q_{1} b_{2}$, 则上式化为

$$
a x_{2}-b y_{2}-c=-s_{2}
$$

同理, 由

可得

$$
\frac{s_{2}}{r_{2}}=b_{3}-\frac{s_{3}}{r_{2}}, \quad s_{3}<r_{2}
$$

$$
s_{3}=r_{2} b_{3}-s_{2}
$$

根据（5）式，可以得到

$$
a p_{2} b_{3}-b q_{2} b_{3}=r_{2} b_{3}
$$


相加（9）与（10）, 可以得到

$$
a\left(x_{2}+p_{2} b_{3}\right)-b\left(y_{2}+q_{2} b_{3}\right)-c=r_{2} b_{3}-s_{2}
$$

若令 $x_{3}=x_{2}+p_{2} b_{3}, y_{3}=y_{2}+q_{2} b_{3}$, 则上式化为

$$
a x_{3}-b y_{3}-c=s_{3}
$$

重复上述步骤, 可以得到

$$
a x_{n}-b y_{n}-c=(-1)^{n+1} s_{n}
$$

其中，

$$
\begin{array}{ll}
x_{n}=x_{n-1}+p_{n-1} b_{n}, & x_{1}=b_{1} \\
y_{n}=y_{n-1}+q_{n-1} b_{n}, & y_{1}=1 \\
s_{n}=r_{n-1} b_{n}-s_{n-1}, & s_{1}=a b_{1}-(b+c)
\end{array}
$$

当 $s_{n}<1$ 时，满足 (11) 式的 $\left(x_{n}, y_{n}\right)$ ，即为不定方程（6）的解。

\section{4 中根元珪的《累约拾遗》}

中根元珪的《累约拾遗》由三个问题和两个应用组成。[6] 这些问题, 都是用一次 同余式的形式给出的。或许因为其最后的两个应用问题与历法有矢, 而当代的和算史 家对此不是很熟悉, 所以, 有爸的和算史研究论述中, 很少仔细地讨论这部著作。[7]

\section{1 《累约拾遗》的问题}

中根元珪《累约拾遗》中设计的三个问题, 反映了一次同余式可能出现的几种不同 的情况, 他们最终都被转化为一种标准的同余形式。

第一问：给定同余式为

$$
75.36 x+240.02 \equiv r^{\prime} \quad(\bmod 301.63)
$$

其中, $0<r^{\prime}<1$ 。令原损 $c=61.61=301.63-240.02$ (原文误为 261.61), 则化为定式 :

$$
75.36 x-c \equiv r \quad(\bmod 301.63)
$$

其中 $0<r<1$ 。这是小数的情形, 余数被限定在单位 1 , 与建部贤弘《累约术》设定的 问题类似。

第二问：给定同余式为

$$
1835 x+2675 \equiv r^{\prime} \quad(\bmod 8400)
$$

其中 $170<r^{\prime}<250$ 。 令原损 $c=5895=170-2675+8400$, 许限 $d=80=250-170$ 。 则化为定式:

$$
1835 x-c \equiv r \quad(\bmod 8400)
$$


其中 $0<r<d_{\text {。 }}$ 于是，问题被化为整数同余式的一般情形。

第三问：给定同余式为

$$
170 x+50 \equiv r^{\prime} \quad(\bmod 952)
$$

其中 $10<r^{\prime}<18$ 。 由于等数 $(170,952)=34$, 所以，上式有解的充分必要条件是： $r^{\prime}=16$ 。令原损 $c=912=10-50+952$, 许限 $d=8=18-10$ 。则化为定式：

$$
170 x-c \equiv r \quad(\bmod 952)
$$

其中 $0<r<d$, 实际上, $r=6$ 。这也是化为整数同余式的情形, 特别之处是, 由于余数 被唯一确定, 因此, 这个同余式的小于模 952 的解也是唯一的。

\section{2 中根元珪的一次同余式的解法}

根据上面的讨论可以看到，中根元珪将余数不定的一次同余的所有问题,都化为标 准型：

$$
a x-c \equiv r \quad(\bmod b)
$$

其中 $a$ 称为累加, $b$ 为累减, 通常, $a<b, c$ 为原损。令许限 $=d$, 余数满足条件 :

$$
0<r<d
$$

为了求解同余式 (12)，先考虑 $c=0$ 的情形:

$$
a x \equiv r \quad(\bmod b)
$$

其中, $a<b, 0<r<d_{\text {。 }}$ 对于这个同余式, 在前面讨论建部贤弘的算法时已经得到了求 解的程序。首先, 按照连分数展开, 可以得到如下的序列 :

$$
\frac{b}{a}=a_{1}+\frac{r_{1}}{a}, \quad \frac{a}{r_{1}}=a_{2}+\frac{r_{2}}{r_{1}}, \quad \frac{r_{1}}{r_{2}}=a_{3}+\frac{r_{3}}{r_{2}}, \quad \cdots, \frac{r_{n-2}}{r_{n-1}}=a+\frac{r_{n}}{r_{n-1}}
$$

由此可以得到一系列的商 $a_{n}$ 与不尽 $r_{n}$ ，以及益段 $p_{n}$ 。因为中根元珪按同余问题处理， 所以，不再考虑损段 $q_{n}$ 。

由此可以将同余式（13）转化为新的同余式

$$
a p_{n} \equiv(-1)^{n} r_{n} \quad(\bmod b)
$$

这个结果非常重要。中根元珪由此出发, 构造同余式（12）的算法程序。首先，由

$$
\frac{k b+c}{a}=b_{1}-\frac{s_{1}}{a}, \quad s_{1}<a, \quad k= \begin{cases}0, & c>a \\ 1, & c<a\end{cases}
$$

可得

$$
a b_{1}-c=s_{1}+k b
$$

若令 $x_{1}=b_{1}$ ，上式可以写成如下的同余形式：

$$
a x_{1}-c \equiv s_{1} \quad(\bmod b)
$$




\begin{tabular}{|c|c|c|c|}
\hline \multicolumn{4}{|c|}{ 干营 } \\
\hline$k$ & 商 $a_{k}$ & 不尽 $r_{k}$ & 谷段 $p_{k}$ \\
\hline 甲1 & $a_{1}$ & $r_{1}=b-a a_{1}$ & $p_{1}=a_{1}$ \\
\hline 乙2 & $a_{2}$ & $r_{2}=a-r_{1} a_{2}$ & $p_{2}=1+p_{1} a_{2}$ \\
\hline 丙 3 & $a_{3}$ & $r_{3}=r_{1}-r_{2} a_{3}$ & $p_{3}=p_{1}+p_{2} a_{3}$ \\
\hline$\cdots$ & $\cdots$ & $\cdots$ & $\cdots$ \\
\hline$n$ & $a_{n}$ & $r_{n}=r_{n-2}-r_{n-1} a_{n}$ & $p_{n}=p_{n-2}+p_{n-1} a_{n}$ \\
\hline \multicolumn{4}{|c|}{ 支营 } \\
\hline & 商 $b_{k}$ & 不尽 $s_{k}$ & 益段 $x_{k}$ \\
\hline 子 1 & $b_{1}$ & $s_{1}=a b_{1}-(b+c)$ & $x_{1}=b_{1}$ \\
\hline 丑2 & $b_{2}$ & $s_{2}=r_{1} b_{2}-s_{1}$ & $x_{2}=x_{1}+p_{1} b_{2}$ \\
\hline 寅3 & $b_{3}$ & $s_{3}=r_{1} b_{3}-s_{2}$ & $x_{3}=x_{2}+p_{2} b_{3}$ \\
\hline$\cdots$ & $\cdots$ & $\cdots$ & $\cdots$ \\
\hline$n$ & $b_{n}$ & $s_{n}=r_{n-1} b_{n}-s_{n-1}$ & $x_{n}=x_{n-1}+p_{n-1} b_{n}$ \\
\hline
\end{tabular}

Table 3. Ganying and Zhiying; 干营与支营

比较 (12) 与 (15), 不难知道, 如果我们可以找到一系列的 $s_{n}$ ，使之不断地缩小，当 $\left|s_{n}\right|>$ $d$ (许限) 时, 恒取 $s_{n}>0$, 直至满足

$$
0<(-1)^{n+1} s_{n}<d
$$

则, 对应的 $x_{n}$ ，就可以满足不定方程（19）。由于序列 $\left\{r_{n}\right\}$ 是逐次递减的，因此，可以利 用序列 $\left\{r_{n}\right\}$ ，来构造 $\left\{s_{n}\right\}$ 。所以，令

$$
\frac{s_{1}}{r_{1}}=b_{2}-\frac{s_{2}}{r_{1}}, \quad s_{2}<r_{1}
$$

可得

$$
s_{2}=r_{1} b_{2}-s_{1}
$$

根据（14）式, 可以得到

$$
a p_{1} b_{2} \equiv-r_{1} b_{2} \quad(\bmod b)
$$

相加（15）与（16）, 可以得到

$$
a\left(x_{1}+p_{1} b_{2}\right)-c \equiv-\left(r_{1} b_{2}-s_{1}\right) \quad(\bmod b)
$$

若令 $x_{2}=x_{1}+p_{1} b_{2}$, 则上式化为

$$
a x_{2}-c \equiv-s_{2} \quad(\bmod b)
$$

同理, 由

$$
\frac{s_{2}}{r_{2}}=b_{3}-\frac{s_{3}}{r_{2}}, \quad s_{3}<r_{2}
$$

可得

$$
s_{3}=r_{2} b_{3}-s_{2}
$$


根据（14）式, 可以得到

$$
a p_{2} b_{3} \equiv r_{2} b_{3} \quad(\bmod b)
$$

相加（17）与 (18)，可以得到

$$
a\left(x_{2}+p_{2} b_{3}\right)-c=r_{2} b_{3}-s_{2} \quad(\bmod b)
$$

若令 $x_{3}=x_{2}+p_{2} b_{3}$, 则上式化为

$$
a x_{3}-c \equiv s_{3} \quad(\bmod b)
$$

重复上述步骤, 可以得到

$$
a x_{n}-c \equiv(-1)^{n+1} s_{n} \quad(\bmod b)
$$

其中，

$$
\begin{aligned}
& x_{n}=x_{n-1}+p_{n-1} b_{n}, \quad x_{1}=b_{1} \\
& s_{n}=r_{n-1} b_{n}-s_{n-1}, \quad s_{1}=a b_{1}-(k b+c), \quad k= \begin{cases}0, & c>a \\
1, & c<a\end{cases}
\end{aligned}
$$

其中 $\left|s_{n}\right|>d$ (许限) 时，总是取 $s_{n}>0$ 。当 $0<(-1)^{n} s_{n}<d$, 满足 (19) 式的 $x_{n}$ 就是 同余式 (12) 的解。不难看出, 中根元珪的算法与建部贤弘的算法, 在构造思想上是一 致的。但是, 由于中根元珪用同余式的形式来处理问题, 不再单独考虑所谓损段 $q_{n}$, 因 此, 简化了相爸的计算。

\section{3 《累约拾遗》的应用题 $\mathrm{I}$}

《累约拾遗》的最后两个问题都是《宣明历》的应用。依笔者之孤陃，似乎未见现 代和算史家对此进行研究, 因此, 让我们稍微做一些讨论。第一个应用问题称：

按建仁二年壬戌岁（1202）冬至, 在庚午日夜子初一刻强, 以辛未为冬至, 盖 为贺朔旦冬至也。由是观之, 每在子初者, 属明日也, 亦不可知。故考贞观 壬午（862）已来常气加时在子初以后者, 如左。（贞观壬午（862）距贞享 甲子 (1684)，行《宣明历》。)

通常我们认为夜牛（子正）为日界，即一日的分界点。中根元珪发现，建仁二年（1202） 冬至时刻在庚午日子初一刻强, 即 $23 \mathrm{~h}$ 至 $24 \mathrm{~h}$ 之间, 而历注当年冬至在辛未日, 即庚午 日之后一天。于是，中根元理试图通过计算证明，对于 24 气的时刻，《宣明历》都是以子 初( $23 \mathrm{~h})$ 为日界的。

令 $t=3068055 / 8400$ (日）表示《宣明历》的回归年常数, $c^{\prime}=4590 / 8400$ (日）为 贞观壬午 (862) 的冬至时刻, 设贞观壬午冬至后第 $x$ 气的时刻为 $r^{\prime}$, 则有

$$
\frac{t}{24} x+c^{\prime} \equiv r^{\prime} \quad(\bmod 1)
$$


我们试图求解 $x$, 使得

$$
\frac{8050}{8400}=\frac{23}{24}<r^{\prime}<1
$$

将《宣明历》一气的长度

$$
\frac{t}{24}=15 \frac{1835+5 / 8}{8400} \text { 日 }
$$

代入上面的同余式, 稍加整理, 可以得到

$$
1835 \frac{5}{8} x-3460 \equiv r \quad(\bmod 8400)
$$

其中：累加 $a=1835+5 / 8$; 累损 $b=8400$; 等数 $(a, b)=1+7 / 8$ 。 原损 $c=3460=$ $8050-4590 ;$ 许限 $d=350=8400-8050$ 。 当不尽 $r=(-1)^{n+1} s_{n}$, 满足

$$
0<r<d
$$

时, 对应的 $x=x_{n}$ ，即为我们所需要的答案。《累约拾遗》中给出的演草如表 4 所示。 因为 $c>a$, 所以,

$$
s_{1}=a b_{1}-c=(1835+5 / 8) \times 2-3460=211+2 / 8
$$

此时, 余数 $r=s_{1}=211+2 / 8<d$, 满足许限 $d$ 设定的条件, 对应的解为 : $x=2$, 即贞 观壬午（862）冬至后第二个气的时刻就发生在 23h-24h 之间。

欲求下一个解, 只需对 $s_{1}$ 加（弱）或减（强）干营的余数 $r_{n}(n \geq 3)$, 只要保证结

\begin{tabular}{|c|c|c|c|c|c|c|c|}
\hline \multicolumn{4}{|c|}{ 干营 } & \multicolumn{4}{|c|}{ 支营 } \\
\hline$n$ & $a_{n}$ & $r_{n}$ & $p_{n}=p_{n-2}+p_{n-1} a_{n}$ & $n$ & $b_{n}$ & $s_{n}$ & $x_{n}$ \\
\hline 甲1 & 4 & 弱 $1057+4 / 8$ & $4=1 \times 4$ & 子 1 & 2 & 强 $211+2 / 8$ & $2=2$ \\
\hline 乙2 & 1 & 强 $778+1 / 8$ & $5=1+4 \times 1$ & & & & \\
\hline 丙3 & 1 & 弱 $279+3 / 8$ & $9=4+5 \times 1$ & & & & \\
\hline 丁 4 & 2 & 强 $19+3 / 8$ & $23=5+9 \times 2$ & & & & \\
\hline 戊5 & 1 & 弱 $60+0 / 8$ & $32=9+23 \times 1$ & & & & \\
\hline
\end{tabular}
果满足 $0<r<d=350$, 则相应的 $x+p_{n}$, 即为所求的结果。例如,

$$
0<r=s_{1}+r_{5}=271+2 / 8<350
$$

所以, $x+p_{5}=2+32=34$, 这个结果的意思是, 贞观壬午 (862) 冬至后第 34 个气的 时刻也发生在 23h-24h 之间。

Table 4. Application 1; 《累约拾遗》应用题 I 演草

\section{4 《累约拾遗》的应用题 II}

《累约拾遗》的第二个应用题，也是有䏌《宣明历》节气的推算。原题称： 
《宣明》求天正经朔入气, 世算历者, 唯言视闰余在大雪定数已下为入大雪, 已上, 为入小雪。而不及言入立冬之数。岂不误乎? 今按国余分二十四万 四千六百七十二已上者, 皆得入立冬。因考贞观壬午（862）已来逢次限者， 如左。[乃起长元五年壬申（1032）至元历元年甲辰（1184，百五十三年之 间，逢此限者，九次。尔后五百年有余，不逢此限，宜乎人不知有此数也。]

天正经朔到冬至时刻的时间, 称为『国余』。由于两个平气的长度（约 30.46 日）大于 一个朔望月的长度 (约 29.53 日), 因此, 天正经朔时刻, 通常在小雪之后。若在小雪与 大雪之间, 称为『入小雪』; 在大雪之后, 称为『入大雪』。但是, 如果采用定气注历, 则 小雪与大雪定气之和：

$$
d^{\prime}=14 \frac{5235+5 / 8}{8400}+14 \frac{4235+5 / 8}{8400} \approx \frac{244672}{8400}<29.53 \text { 日 }
$$

也就是说, 天正经朔时刻, 有可能出现在立冬与小雪之间, 称为『入立冬』。我们可以建 立如下的同余式, 来计算贞观壬午 (862) 之后第 $x$ 年的国余, 即当年的天正经朔时刻 到冬至时刻的时间 $r^{\prime}$ :

$$
t x+c^{\prime} \equiv r^{\prime} \quad(\bmod u)
$$

其中《宣明历》的常数：回归年 $t=3068055 / 8400$ (日) ; 朔望月 $u=248057 / 8400$ （日）； $c^{\prime}=94249 / 8400$ (日), 表示贞观壬午 (862) 国余, 称为『元数』。因此, 当余数 $r^{\prime}$ 满足

$$
d^{\prime}<r^{\prime}<u
$$

时, 天正经朔时刻应该在立冬与小雪之间，即入立冬。同余式（20）可以简化为

$$
(t-12 u) x+c^{\prime} \equiv r^{\prime} \quad(\bmod u)
$$

其中, $t-12 u=91371 / 8400$ (日)。以 8400 同乘于上面的同余式, 并秒加整理, 可以得 到同余式

$$
91371 x-150423 \equiv r \quad(\bmod 248057)
$$

其中：累加 $a=91371$; 累损 $b=248057$; 等数 $(a, b)=1$ 。原损 $c=150423=$ $244672-94249$ ；许限 $d=3385=248057-244672$ 。当不尽 $r=(-1)^{n+1} s_{n}$ ，满足

$$
0<r<d
$$

时, 对应的 $x=x_{n}$ ，即为我们需要的答案。《累约拾遗》给出的演草如表 5 所示。其中

$$
s_{6}=r_{5} b_{6}-s_{5}=350 \times 7-5563=-3113
$$

所以, 余数 $r=-s_{6}=3113<d$, 这是满足许限 $d$ 的最大的余数。相应的解为 $x=x_{7}=$ 170 , 即贞观壬午 (862) 之后第 170 年的天正经朔时刻一定在立冬与小雪之间, 这一年 是长元五年 (1032)。求下一个解的方法, 与上一节讨论的应用题 I 类似。 


\begin{tabular}{|c|c|c|c|c|c|c|c|}
\hline \multicolumn{4}{|c|}{ 干营 } & \multicolumn{4}{|c|}{ 支营 } \\
\hline$n$ & $a_{n}$ & $r_{n}$ & $p_{n}=p_{n-2}+p_{n-1} a_{n}$ & $n$ & $b_{n}$ & $s_{n}$ & $x_{n}=x_{n-1}+p_{n-1} b_{n}$ \\
\hline 甲1 & 2 & 弱 65315 & $2=1 \times 2$ & 子 1 & 2 & 强32319 & $2=2$ \\
\hline 乙2 & 1 & 强26056 & $3=1+2 \times 1$ & 丑2 & 1 & 弱32996 & $4=2+2 \times 1$ \\
\hline 丙3 & 2 & 弱13203 & $8=2+3 \times 2$ & 寅3 & 2 & 强19116 & $10=4+3 \times 2$ \\
\hline 丁 4 & 1 & 强12853 & $11=3+8 \times 1$ & 卯4 & 2 & 弱7290 & $26=10+8 \times 2$ \\
\hline 戊5 & 1 & 弱350 & $19=8+11 \times 1$ & 辰5 & 1 & 强55634 & $37=26+11 \times 1$ \\
\hline 己6 & 28 & 强3053 & $543=11+19 \times 28$ & 巳6 & 7 & 强3113 & $170=37+19 \times 7$ \\
\hline 己6 & 29 & 强2703 & $562=11+19 \times 29$ & & & & \\
\hline
\end{tabular}

Table 5. Application 2 in Ruiyaku Shui; 《累约拾遗》应用题 II 演草

\section{5 中日不定分析的异同}

建部贤弘和中根元珪的累约术, 分别给出了一次不定方程与同余式的算法, 从算法 的构造思想来看, 两者是相通的。建部贤弘考虑了一般形式的二元一次不定方程：

$$
a x-b y-c=r, \quad|r|<1
$$

中根元珪考虑了对应的一次同余问题：

$$
a x-c \equiv r \quad(\bmod b), \quad 0<r<d
$$

在构造上述问题的解法时，都采用了如下的策略：先考虑 $c=0$ 时的算法，并由此出发， 得出一般问题的解法程序。值得一提的是, 建部贤弘在处理 $c=0$ 时的不定方程的时 候, 应用了连分数展开的算法, 并得到了渐近分数列。

中国古代除了百鸡之类的问题以外，很少从不定方程的角度设计问题。通常都将 问题化为同余式来处理。在解决同余式的时候, 矢键的算法是大衍求一术, 也就是建部 贤弘与中根元珪设计的求㿽段 $p_{n}$ 的程序，一直进行到 $(-1)^{n} r_{n}=1$ 。这个算法中，并未 涉及损率 $q_{n}$ ，因此，累约术对于连分数算法的应用，是建部贤弘的一项创造。

中根元珪选取的应用问题, 都是《宣明历》带来的实际问题, 与中国古代利用演纪 术推求上元积年的问题类似。利用同余式处理不定方程, 可以大大简化算法的复杂性， 这是中根元珪对建部贤弘之累约术的发展。

当同余式中余数 $r$ 的选择范围不是很大的时候, 利用大衍求一术, 直接求得乘 率 $k=p_{n}$ ，使得 $(-1)^{n} r_{n}=1$ ，此时

$$
a k \equiv 1 \quad(\bmod b)
$$

由此可以得到同余式（21）的解

$$
x \equiv(r+c) k \quad(\bmod b)
$$

这是中国古代处理此类问题的一般方法。不过, 当余数 $r$ 的选择范围很大时, 欲根据 
（22）式得到合适的解, 就有点费事。此时, 建部贤弘与中根元珪设计的支营的算法, 就 显得很有必要了, 这也是和算家对一次不定分析算法的另一项创造。

由于累约术处理的问题, 大体上都是单个的不定方程或同余式, 因此, 与中国剩余 定理基本上没有䏌系。实际上, 中国剩余定理是秦九韶的一项数学创造, 它的诞生, 虽 然与历法上元积年的推求有爷, 但是, 却并未真正在历法计算上发挥作用。[4,24-34]

另外, 值得指出的是, 建部贤弘与中根元珪的累约术, 都是非常完整的机械化的程 序算法。这一点,继承了中国古代处理不定分析问题的算法传统。

\section{6 结论}

本文以建部贤弘和中根元珪的累约术为例, 讨论了和算家研究这些问题的出发点， 研究方式, 及其与中国古代数学家的异同之处。根据上面的讨论可以看出, 和算家的出 发点, 都是基于中国传统数理天文学所提出的问题,他们处理这些问题的思想方法与中 国古代类似，也是通过设计机械化的程序算法，获得问题的解答。

值得我们深思的是，中国传统的数学家，绝大部分都是带着直接为历法服务的功利 性的目的来设计算法的，因此，他们的数学创造，常常是有局限的、适可而止的。

与之形成鲜明对比的是, 和算家通常都是比较纯粹的数学家, 因此, 他们的研究较少 急功近利的、实用主义的桎梏, 导致他们在探索中国传统数学问题的处理方法时, 更加 矢注解决这些问题的算法的数学本质, 从而将现实问题抽象为更为一般的数学问题。

例如, 对于一次不定分析, 建部贤弘总是将问题抽象为如下的数学形式：

$$
a x-b y-c=r, \quad|r|<1
$$

其中 $0<a<b, c$ 为任意给定的有理数。而中根元珃则将问题归结为统一的同余形式：

$$
a x-c \equiv r \quad(\bmod b), \quad 0<r<d
$$

其中 $a, b, c, d$ 都是非负有理数。他们都是从 $c=0$ 的特殊情形出发, 构造出上述问题的 一般算法程序。

中算家在设计算法的时候，常常从具体问题出发，依赖于其几何意义的阐述，较少 运用代数变换的技巧，因此，不容易洞悉他们所设计的算法的数学本质，由此便很难抽 象、归纳出更加一般的程序算法。这一点, 中算家的内插法是一个典型的事例：对于 中国古代历法家来说, 从使用功能的角度看, 基于几何图形构造的分段二次内插法（刘 焯,600)，或三次内插法（郭守敬,1280)，不仅是合理的，而且是充分的。很难，也似乎没 有必要, 在此基础上做出新的创造。[5] 作为一个纯粹的数学家, 秦九韶在《数书九章》 中所设计的中国剩余定理和大衍求一术, 都成功地超越了实际问题的限制。可惜, 这样 的中国数学家并不多见。 
将现实问题, 抽象为数学问题, 是近代科学的一个极为重要的标志。和算家在现实 中提取问题, 并能超越具体问题的束缚, 是和算家在继承中算传统时, 能够做出更多深 刻的、创造性的数学成就的主要原因, 这也是和算对中国数学传统的最为重要的创新。

吴文俊先生认为, 在数学历史的长河中, 应该存在着两种交互出现的数学潮流, 其 一为公理化的逻辑演绎体系, 其二为机械化的程序算法体系。后者的典型代表, 就是中 国传统数学。对于数学史来说, 以算法为特征的中国古典数学, 是否可以得到深刻而全 面的发展, 并在适当时机成为数学发展的主流, 是一个引人矢注的问题。[9]

和算中的许多问题, 来源于中国传统的数学与数理天文学, 比较 17 世纪以来中日 两国数学家在数学领域的继承与创新, 是非常有趣的题目。

中国古代数学,在 14 世纪以后, 基本上停止了发展。在 17 世纪（明末清初）西方 科学第一次传入中国的时候，一些数学家开始树立复兴传统数学的旗帜。但是，从清代 数学家的表现来看, 在继承并发扬中国数学传统方面, 似乎并不成功, 我们很少看到清 代的数学家在机械化算法的设计方面, 取得令人赞叹的成就。

与此同时, 日本明治之前的数学 (和算), 继承了中国古代数学的传统, 并且有深刻 的发展和创新。和算家的数学实践证明, 中国传统数学的思想方法, 在代数变换技巧的 辅助下，可以在很大程度上，发展出一套完美的机械化的程序算法体系。只要将合适的 现实问题, 转化为合适的数学问题, 机械化的程序算法体系是可以大有作为的。

吴文俊先生自己在数学机械化方面的工作, 是现代数学家继承中国传统数学的一 次实践。不过，我们确实希望在没有广泛经受西方近代数学侵淫的文化中，可以找到中 国传统数学获得继承和发展的实例。

如果说, 在人类数学文明的历史上, 以机械化算法为特色的数学思想, 确曾主导过 数学的潮流, 并在计算机的时代, 有可能再度成为数学家矢注的焦点, 那么, 和算家的数 学活动, 作为中国数学传统得以延续和发展的一个珍贵的化石, 或许可以算是我们必须 认真对待和算研究的一个重要的理由。

参考文献

1. Hua Luogeng, A Story on the ratio of a circle's circumference to its diameter by $\mathrm{Zu}$ Chongzhi, Hua Luogeng's collection of scientific works, Shanghai: Shanghai Jiaoyu Publishing Co., 1984, 47-80. 华罗庚, 从租冲之的圆周率谈起, 华罗庚科普著作选集, 上海: 上海 教育出版社, 1984, 47-80.

2. Li Jimin, Interpretation of "TongGiLu", History of Chinese Mathematics Lunwen Ji, vol. 1, Jinan: Shandong Jiaoyu Publishing Co., 1985, 24-36. 李继闵，“通其率”考释，中国数学 史论文集 (1), 济南 : 山东教育出版社, 1985, 24-36. 
3. The Japan Academy ed., A History of Japanese Mathematics before Meiji Period, vol. 2, Tokyo: Linchuan Bookstore, 1979, 310-318. 日本学士院编，「明治前日本数学史 (2)」，东京: 临川 书店, 1979, 310-318.

4. Qu Anjing, Chinese Astronomy and Mathematics, Beijing, Science Publishing Co., 2005. 曲 安京, 中国历法与数学, 北京, 科学出版社, 2005, 74-91.

5. Qu Anjing, "The Succession and Innovation of Wasan to Chinese Mathematics -A case study on Seki's interpolation", Journal for History of Mathematics 26(4) (2013), 219-232. 曲 安京. 和算对中算的继承与创新一以爸孝和的内插法为例. Journal for History of Mathematics 26(4) (2013), 219-232.

6. Takebe Katahiro, The Method of Successive Divisions, possession of Tohoku University Library. 建部贤弘, 累约术, (日本) 东北大学图书馆藏, 冈本文库写 0304.

7. Tamotsu Tsuchikura, The Method of Successive Divisions by Takebe Katahiro and Nakane Genkei, Knobloch, Komatsu, Liu (ed.). Seki, Founder of Modern Mathematics in Japan. Tokyo: Springer, 2013, 343-352.

8. Tsuchikura, et al. ed., Invitation to History of Mathematics in East Asia-Collected Works of Fujiwara Matsusaburo on the History of Mathematics, Sendai: Tohoku University Publishing House, 2007, 21-30. 土倉保等编, 「東洋数学史一の招待一藤原松三郎数学史論文集」，仙台 : 東北大学出版会, 2007, 21-30.

9. Wu Wentsun, Mathematics Mechanization, Beijing: Science Press \& Dordrecht: Kluwer Academic Publishers, 2000, 1-66. 